\title{
Remote Preparation of the Two-Particle State
}

\author{
YAN Feng-Li, ZHANG Guo-Hua \\ College of Physics Science and Information Engineering, \\ Hebei Normal University, Shijiazhuang 050016, China
}

\begin{abstract}
We present a scheme of remote preparation of the two-particle state by using two EinsteinPodolsky-Rosen pairs or two partial entangled two-particle states as the quantum channel. The probability of the successful remote state preparation is obtained.

PACS numbers: 03.65.Ta, 03.67.Hk, 03.67.Lx
\end{abstract}

Quantum entanglement is a valuable resource for the implementation of quantum computation and quantum communication protocols, like quantum teleportation $[1,2]$, quantum key distribution [3,4], quantum secure direct communication [5], dense coding [6-8], quantum computation [9], remote state preparation [10-15] and so on. Quantum teleportation is regarded as one of the most profound results of quantum information theory. In the original quantum teleportation protocol of Bennett et al. [1], it was showed that an unknown state of a qubit can be perfectly transported from a sender Alice to a remote receiver Bob with the aid of long-range Einstein-Podolsky-Rosen correlation and transmission of two bits of classical information without transmission of the carrier of the quantum state. They have also generalized the protocol for an unknown qubit by using a maximally entangled state in $d \times d$ dimensional Hilbert space and sending $2 \log _{2} d$ bits of classical information.

Another important application of quantum entanglement is remote state preparation, where two spatially distant people Alice and Bob can prepare an quantum state known to Alice but unknown to Bob, with the aid of previously shared quantum entanglement and the classical communication. Recently, Pati [10] presented a protocol of remotely preparing a special ensemble of states. Lo [11] showed that remote state preparation requires less classical communication than teleportation for the special ensembles of states, but for general states the classical communication cost of teleportation would be equal to that of remote state preparation. Bennett et al. [12] studied the trade off between entanglement cost and classical communication cost in remote state preparation. Since then, some people have investigated various theoretical protocols about generalization of remote state preparation. Zhan [13] gave a scheme for preparing remotely a three-particle GHZ state. Huang et al. [14] put forward a protocol for preparing remotely the multipartite pure state. A scheme for preparing remotely a two-particle entangled state via two pairs of entangled particles was presented by Liu et al. [15].

In this paper, we propose a scheme of preparing remotely the two-particle state. Two Einstein-PodolskyRosen pairs and two partial entangled two-particle states as the quantum channel are considered, respectively. The probability of the successful remote state preparation is calculated.

Let us first begin our remote state preparation with two Einstein-Podolsky-Rosen pairs as the quantum channel. We suppose that a sender Alice wants to help a remote receiver Bob to prepare a two-particle state in the following formation

$$
|\phi\rangle=\alpha|00\rangle+\beta|01\rangle+\gamma|10\rangle+\delta|11\rangle,
$$

where $\alpha$ and $\gamma$ are real numbers, $\beta$ and $\delta$ are complex numbers and $\beta^{*} \delta$ is real, $|\alpha|^{2}+|\beta|^{2}+|\gamma|^{2}+|\delta|^{2}=1$. We suppose that Alice knows $\alpha, \beta, \gamma$ and $\delta$ completely, but Bob does not know them at all. We also assume that the quantum channel shared by Alice and Bob is composed of two Einstein-Podolsky-Rosen pairs

$$
\begin{aligned}
& |\psi\rangle_{12}=\frac{1}{\sqrt{2}}(|00\rangle+|11\rangle)_{12}, \\
& |\psi\rangle_{34}=\frac{1}{\sqrt{2}}(|00\rangle+|11\rangle)_{34},
\end{aligned}
$$

where particles 1 and 3 belong to Alice while Bob has particles 2 and 4 . In order to help Bob to remotely prepare a two-particle state stated in Eq.(1) on the particles 2 and 4, Alice must make a measurement on her two particles 1 and 3 . The measurement basis chosen by Alice is a set of mutually orthogonal basis vectors $\left\{|\varphi\rangle_{13},\left|\varphi_{\perp}\right\rangle_{13},|\psi\rangle_{13},\left|\psi_{\perp}\right\rangle_{13}\right\}$, where

$$
\begin{aligned}
& |\varphi\rangle_{13}=(\alpha|00\rangle+\beta|01\rangle+\gamma|10\rangle+\delta|11\rangle)_{13} \\
& \left|\varphi_{\perp}\right\rangle_{13}=\left(-\delta^{*}|00\rangle+\gamma|01\rangle-\beta^{*}|10\rangle+\alpha|11\rangle\right)_{13} \\
& |\psi\rangle_{13}=(\gamma|00\rangle+\delta|01\rangle-\alpha|10\rangle-\beta|11\rangle)_{13} \\
& \left|\psi_{\perp}\right\rangle_{13}=\left(\beta^{*}|00\rangle-\alpha|01\rangle-\delta^{*}|10\rangle+\gamma|11\rangle\right)_{13}
\end{aligned}
$$

Here $\left\{|00\rangle_{13},|01\rangle_{13},|10\rangle_{13},|11\rangle_{13}\right\}$ is the computation basis. Then we have

$$
\begin{aligned}
& |\psi\rangle_{12} \otimes|\psi\rangle_{34} \\
= & \frac{1}{2}\left[|\varphi\rangle_{13}\left(\alpha|00\rangle+\beta^{*}|01\rangle+\gamma|10\rangle+\delta^{*}|11\rangle\right)_{24}\right. \\
& +\left|\varphi_{\perp}\right\rangle_{13}(-\delta|00\rangle+\gamma|01\rangle-\beta|10\rangle+\alpha|11\rangle)_{24} \\
& +|\psi\rangle_{13}\left(\gamma|00\rangle+\delta^{*}|01\rangle-\alpha|10\rangle-\beta^{*}|11\rangle\right)_{24} \\
& \left.+\left|\psi_{\perp}\right\rangle_{13}(\beta|00\rangle-\alpha|01\rangle-\delta|10\rangle+\gamma|11\rangle)_{24}\right] .
\end{aligned}
$$


Thus if Alice performs a measurement in the basis $\left\{|\varphi\rangle_{13},\left|\varphi_{\perp}\right\rangle_{13},|\psi\rangle_{13},\left|\psi_{\perp}\right\rangle_{13}\right\}$ on her two particles 1 and 3, then each outcome will occur with the equal probability $\frac{1}{4}$. If Alice's measurement result is $\left|\varphi_{\perp}\right\rangle$, then particles 2 and 4 will collapse into the state

$$
(-\delta|00\rangle+\gamma|01\rangle-\beta|10\rangle+\alpha|11\rangle)_{24}
$$

After that if Alice communicates to Bob of her actual measurement outcome via a classical channel, then Bob will be able to apply the following unitary transformation

$$
U=(|0\rangle\langle 1|+| 1\rangle\langle 0|)_{2} \otimes(|0\rangle\langle 1|-| 1\rangle\langle 0|)_{4}
$$

on his particles 2 and 4 . The resulting state of Bob's particles will be the original state $|\phi\rangle$.

Likewise if the actual result of Alice's measurement is $\left|\psi_{\perp}\right\rangle_{13}$, then Bob gets the state

$$
(\beta|00\rangle-\alpha|01\rangle-\delta|10\rangle+\gamma|11\rangle)_{24}
$$

When Bob received the classical information of the actual measurement result sent by Alice, he can perform an appropriate operation

$$
U^{\prime}=(|0\rangle\langle 0|-| 1\rangle\langle 1|)_{2} \otimes(|0\rangle\langle 1|-| 1\rangle\langle 0|)_{4}
$$

on his particles 2 and 4 to obtain the state $|\phi\rangle$. So when these two measurement outcomes happen, Alice can help Bob to remotely prepare the two-particle state $|\phi\rangle$.

However, when the measurement outcome is $|\varphi\rangle_{13}$ $\left(|\psi\rangle_{13}\right)$, the remote state preparation can not be successful, as the state of the particles 2 and 4 will be $\left(\alpha|00\rangle+\beta^{*}|01\rangle+\gamma|10\rangle+\delta^{*}|11\rangle\right)_{24}\left(\left(\gamma|00\rangle+\delta^{*}|01\rangle-\right.\right.$ $\left.\left.\alpha|10\rangle-\beta^{*}|11\rangle\right)_{24}\right)$. Because Bob does not know the coefficients $\alpha, \beta, \gamma$ and $\delta$ at all, he cannot transform either the state $\left(\alpha|00\rangle+\beta^{*}|01\rangle+\gamma|10\rangle+\delta^{*}|11\rangle\right)_{24}$ or $\left(\gamma|00\rangle+\delta^{*}|01\rangle-\alpha|10\rangle-\beta^{*}|11\rangle\right)_{24}$ into the state $|\phi\rangle$. But, if $\alpha, \beta, \gamma$ and $\delta$ are real numbers, the situation would be changed. When Alice's measurement result $|\varphi\rangle_{13}$ or $|\psi\rangle_{13}$ occurs, it is not difficult for Bob to prepare the two-particle state $|\phi\rangle$ by performing the suitable unitary operation determined by the outcome of Alice's measurement. Here we omit the concrete steps.

Now, we present a scheme for preparing remotely a two-particle state via two non-maximally entangled states. Suppose that Alice still wishes to help Bob to prepare remotely the state $|\phi\rangle$ in Eq.(1), but the two entangled states shared by Alice and Bob are two nonmaximally entangled states

$$
\begin{aligned}
& |\psi\rangle_{12}=(a|00\rangle+b|11\rangle)_{12}, \\
& |\psi\rangle_{34}=(c|00\rangle+d|11\rangle)_{34},
\end{aligned}
$$

where the parameters $a, b, c$ and $d$ are real numbers, $|a|^{2}+|b|^{2}=1,|c|^{2}+|d|^{2}=1$, and $|a| \leq|b|,|c| \leq|d|$. We also assume that the particles 1 and 3 belong to Alice while Bob has particles 2 and 4. Since Alice knows the parameters $\alpha, \beta, \gamma$ and $\delta$ exactly, she can perform a measurement on particles 1 and 3 in the basis $\left\{|\varphi\rangle_{13},\left|\varphi_{\perp}\right\rangle_{13},|\psi\rangle_{13},\left|\psi_{\perp}\right\rangle_{13}\right\}$. A simple algebraic rearrangement of the expression $|\psi\rangle_{12} \otimes|\psi\rangle_{34}$ in terms of the states $|\varphi\rangle_{13},\left|\varphi_{\perp}\right\rangle_{13},|\psi\rangle_{13},\left|\psi_{\perp}\right\rangle_{13}$ yields

$$
\begin{aligned}
& |\psi\rangle_{12} \otimes|\psi\rangle_{34} \\
= & |\varphi\rangle_{13}\left(a c \alpha|00\rangle+a d \beta^{*}|01\rangle+b c \gamma|10\rangle+b d \delta^{*}|11\rangle\right)_{24} \\
& +\left|\varphi_{\perp}\right\rangle_{13}(-a c \delta|00\rangle+a d \gamma|01\rangle-b c \beta|10\rangle+b d \alpha|11\rangle)_{24} \\
& +|\psi\rangle_{13}\left(a c \gamma|00\rangle+a d \delta^{*}|01\rangle-b c \alpha|10\rangle-b d \beta^{*}|11\rangle\right)_{24} \\
& +\left|\psi_{\perp}\right\rangle_{13}(a c \beta|00\rangle-a d \alpha|01\rangle-b c \delta|10\rangle+b d \gamma|11\rangle)_{24} .
\end{aligned}
$$

Therefore, if the actual result of Alice's measurement on the two particles 1 and 3 is $\left|\varphi_{\perp}\right\rangle_{13}$ with the probability $|b d \alpha|^{2}+|b c \beta|^{2}+|a d \gamma|^{2}+|a c \delta|^{2}$ then the state of particles 2 and 4 will be

$$
|\varphi\rangle_{24}=\frac{(-a c \delta|00\rangle+a d \gamma|01\rangle-b c \beta|10\rangle+b d \alpha|11\rangle)_{24}}{\sqrt{|b d \alpha|^{2}+|b c \beta|^{2}+|a d \gamma|^{2}+|a c \delta|^{2}}}
$$

When Bob is informed the actual measurement outcome $\left|\varphi_{\perp}\right\rangle_{13}$ by Alice via a classical channel, he can get the original state described in Eq.(1) with certain probability.

Firstly, Bob operates a unitary operation

$$
U_{1}=(|0\rangle\langle 1|+| 1\rangle\langle 0|)_{2} \otimes(|0\rangle\langle 1|-| 1\rangle\langle 0|)_{4}
$$

on particles 2 and 4 . Obviously $U_{1}$ will transform the state $|\varphi\rangle_{24}$ into

$$
\left|\varphi^{\prime}\right\rangle_{24}=\frac{(b d \alpha|00\rangle+b c \beta|01\rangle+a d \gamma|10\rangle+a c \delta|11\rangle)_{24}}{\sqrt{|b d \alpha|^{2}+|b c \beta|^{2}+|a d \gamma|^{2}+|a c \delta|^{2}}}
$$

Secondly, Bob introduces an auxiliary two-level particle $a$ with the initial state $|0\rangle_{a}$ and performs a collective unitary transformation

$$
U_{2}=\left(\begin{array}{cccccccc}
\frac{a c}{b d} & A & 0 & 0 & 0 & 0 & 0 & 0 \\
A & -\frac{a c}{b d} & 0 & 0 & 0 & 0 & 0 & 0 \\
0 & 0 & \frac{a}{b} & B & 0 & 0 & 0 & 0 \\
0 & 0 & B & -\frac{a}{b} & 0 & 0 & 0 & 0 \\
0 & 0 & 0 & 0 & \frac{c}{d} & C & 0 & 0 \\
0 & 0 & 0 & 0 & C & -\frac{c}{d} & 0 & 0 \\
0 & 0 & 0 & 0 & 0 & 0 & 1 & 0 \\
0 & 0 & 0 & 0 & 0 & 0 & 0 & -1
\end{array}\right)
$$

on particles 2, 4 and $a$ under the basis $\left\{|000\rangle_{24 a},|001\rangle_{24 a},|010\rangle_{24 a},|011\rangle_{24 a},|100\rangle_{24 a},|101\rangle_{24 a}\right.$, $\left.|110\rangle_{24 a},|111\rangle_{24 a}\right\}$, where

$$
A=\sqrt{1-\left(\frac{a c}{b d}\right)^{2}}, \quad B=\sqrt{1-\left(\frac{a}{b}\right)^{2}}, C=\sqrt{1-\left(\frac{c}{d}\right)^{2}} .
$$

Since it has been assumed that $|a| \leq|b|$ and $|c| \leq|d|$, so one has $|a c|^{2} \leq|b d|^{2}$. The unitary transformation $U_{2}$ 
will transform $\left|\varphi^{\prime}\right\rangle_{24}|0\rangle_{a}$ into

$$
\begin{aligned}
\left|\varphi^{\prime \prime}\right\rangle_{24 a}= & \frac{1}{\sqrt{|b d \alpha|^{2}+|b c \beta|^{2}+|a d \gamma|^{2}+|a c \delta|^{2}}} \\
& {\left[a c(\alpha|00\rangle+\beta|01\rangle+\gamma|10\rangle+\delta|11\rangle)_{24}|0\rangle_{a}\right.} \\
& +\left(\alpha \sqrt{(b d)^{2}-(a c)^{2}}|00\rangle+c \beta \sqrt{b^{2}-a^{2}}|01\rangle\right. \\
& \left.\left.+a \gamma \sqrt{d^{2}-c^{2}}|10\rangle\right)_{24}|1\rangle_{a}\right] .
\end{aligned}
$$

Finally, Bob performs a measurement on auxiliary particle $a$ in the basis $\left\{|0\rangle_{a},|1\rangle_{a}\right\}$. If the result of his measurement is $|1\rangle_{a}$, then the remote preparation of the original state fails. If the measurement outcome $|0\rangle_{a}$ occurs with probability $\frac{|a c|^{2}}{|b d \alpha|^{2}+|b c \beta|^{2}+|a d \gamma|^{2}+|a c \delta|^{2}}$, then the remote preparation of a two-particle state $|\phi\rangle$ is successfully realized. Evidently, when actual measurement outcome $\left|\varphi_{\perp}\right\rangle_{13}$ is obtained, the probability of successfully remote state preparation is $\left(|b d \alpha|^{2}+|b c \beta|^{2}+|a d \gamma|^{2}+\right.$ $\left.|a c \delta|^{2}\right) \frac{|a c|^{2}}{|b d \alpha|^{2}+|b c \beta|^{2}+|a d \gamma|^{2}+|a c \delta|^{2}}=|a c|^{2}$.

Similarly, by Eq.(12), if Alice's measurement result on particles 1 and 3 is $\left|\psi_{\perp}\right\rangle_{13}$ with the probability $|a d \alpha|^{2}+$ $|a c \beta|^{2}+|b d \gamma|^{2}+|b c \delta|^{2}$, the state of particles 2 and 4 will become

$$
|\psi\rangle_{24}=\frac{(a c \beta|00\rangle-a d \alpha|01\rangle-b c \delta|10\rangle+b d \gamma|11\rangle)_{24}}{\sqrt{|a d \alpha|^{2}+|a c \beta|^{2}+|b d \gamma|^{2}+|b c \delta|^{2}}} .
$$

Now Bob operates the following unitary transformation

$$
U_{1}^{\prime}=(|0\rangle\langle 0|-| 1\rangle\langle 1|)_{2} \otimes(-|0\rangle\langle 1|+| 1\rangle\langle 0|)_{4}
$$

on particles 2 and 4 . Hence the state shown in Eq.(18) was transformed into

$$
\left|\psi^{\prime}\right\rangle_{24}=\frac{(a d \alpha|00\rangle+a c \beta|01\rangle+b d \gamma|10\rangle+b c \delta|11\rangle)_{24}}{\sqrt{|a d \alpha|^{2}+|a c \beta|^{2}+|b d \gamma|^{2}+|b c \delta|^{2}}} .
$$

Next, Bob introduces an auxiliary particle $a$ with the initial state $|0\rangle_{a}$ and performs a unitary transformation

$$
U_{2}^{\prime}=\left(\begin{array}{cccccccc}
\frac{c}{d} & C & 0 & 0 & & 0 & 0 & 0 \\
C & -\frac{c}{d} & 0 & 0 & 0 & 0 & 0 & 0 \\
0 & 0 & 1 & 0 & 0 & 0 & 0 & 0 \\
0 & 0 & 0 & -1 & 0 & 0 & 0 & 0 \\
0 & 0 & 0 & 0 & \frac{a c}{b d} & A & 0 & 0 \\
0 & 0 & 0 & 0 & A & -\frac{a c}{b d} & 0 & 0 \\
0 & 0 & 0 & 0 & 0 & 0 & \frac{a}{b} & B \\
0 & 0 & 0 & 0 & 0 & 0 & B & -\frac{a}{b}
\end{array}\right)
$$

on particles 2, 4 and $a$ under the basis $\left\{|000\rangle_{24 a},|001\rangle_{24 a}\right.$, $\left.|010\rangle_{24 a},|011\rangle_{24 a},|100\rangle_{24 a},|101\rangle_{24 a},|110\rangle_{24 a},|111\rangle_{24 a}\right\}$. It is straightforward to verify that the resulting state of of particles 2, 4 and $a$ is

$$
\begin{aligned}
\left|\psi^{\prime \prime}\right\rangle_{24 a}= & \frac{1}{\sqrt{|a d \alpha|^{2}+|a c \beta|^{2}+|b d \gamma|^{2}+|b c \delta|^{2}}} \\
& {\left[a c(\alpha|00\rangle+\beta|01\rangle+\gamma|10\rangle+\delta|11\rangle)_{24}|0\rangle_{a}\right.} \\
& +\left(a \alpha \sqrt{d^{2}-c^{2}}|00\rangle+\gamma \sqrt{(b d)^{2}-(a c)^{2}}|10\rangle\right. \\
& \left.\left.+c \delta \sqrt{b^{2}-a^{2}}|11\rangle\right)_{24}|1\rangle_{a}\right] .
\end{aligned}
$$

The above equation shows that Bob can construct a twoparticle state, which Alice wishes to prepare remotely, with certain probability by performing a measurement on auxiliary particle $a$ in the basis $\left\{|0\rangle_{a},|1\rangle_{a}\right\}$. If Bob's actual measurement result is $|0\rangle_{a}$, then remote state preparation is successful; otherwise remote state preparation fails. It is easy to prove that the successful probability of remote state preparation in this case is $\left(|a d \alpha|^{2}+|a c \beta|^{2}+\right.$ $\left.|b d \gamma|^{2}+|b c \delta|^{2}\right) \frac{|a c|^{2}}{|a d \alpha|^{2}+|a c \beta|^{2}+|b d \gamma|^{2}+|b c \delta|^{2}}=|a c|^{2}$.

However, if the Alice's actual measurement outcome on particles 1 and 3 is $|\varphi\rangle_{13}\left(|\psi\rangle_{13}\right)$, Bob will obtain the state $\left(a c \alpha|00\rangle+a d \beta^{*}|01\rangle+b c \gamma|10\rangle+b d \delta^{*}|11\rangle\right)_{24}$ $\left(\left(a c \gamma|00\rangle+a d \delta^{*}|01\rangle-b c \alpha|10\rangle-b d \beta^{*}|11\rangle\right)_{24}\right)$. Since Bob has no knowledge of these states, he can not unitary convert each of them into the original state, so remote state preparation fails. But, when $\alpha, \beta, \gamma$ and $\delta$ are real numbers, the situation is not the same. In this case Bob can prepare the state in every Alice's measurement outcome with certain probability. For saving space we omit the concrete steps for preparing.

Synthesizing two cases (the Alice's the actual measurement outcome is either $\left|\varphi_{\perp}\right\rangle_{13}$ or $\left.\left|\psi_{\perp}\right\rangle_{13}\right)$ the probability of the successful remote state preparation is $2|a c|^{2}$. If $|a|=|b|=|c|=|d|=\frac{1}{\sqrt{2}}$, namely, the quantum channel consists of two Einstein-Podolsky-Rosen pairs, the probability equals to $50 \%$.

In summary, we have presented a scheme of the remote preparation of a two-particle state via two EinsteinPodolsky-Rosen pairs and two partial entangled twoparticle state, respectively. The two-particle state can be prepared probabilistically if the sender performs a measurement and the receiver introduces an appropriate unitary transformation with the aid of long-range EinsteinPodolsky-Rosen correlation and transmission of two bits of classical information. Furthermore we obtained the probability of the successful remote state preparation of the two-particle state.

\section{Acknowledgments}

This work was supported by the National Natural Science Foundation of China under Grant No: 10671054 and Hebei Natural Science Foundation of China under Grant Nos: A2005000140;07M006, and the Key Project of Science and Technology Research of Education Ministry of China under Grant No:207011. 
[1] Bennett C H, Brassard G, Crepeau C, Jozsa R, Peres A, and Wootters W K 1993 Phys. Rev. Lett. 701895

[2] Gao T, Wang Z X and Yan F L 2003 Chin. Phys. Lett. 202094

[3] Ekert A K 1991 Phys. Rev. Lett. 67661

[4] Wang X B, Hiroshima T, Tomita A and Hayashi M 2007 Phys. Rep. 4481

[5] Long G L, Deng F G, Wang C, Li X H, Wen K and Wang W Y 2007 Front. Phys. China, 2251

[6] Bennett C H and Wiesner S J 1992 Phys. Rev. Lett. 69 2881

[7] Wang M Y, Yang L G and Yan F L 2005 Chin. Phys.
Lett. 221053

[8] Yan F L, Wang M Y 2004 Chin. Phys. Lett. 211195

[9] Barenco A 1996 Contemp. Phys. 37375

[10] Pati A K 2000 Phys. Rev. A 63014302

[11] Lo H K 2000 Phys. Rev. A 62012313

[12] Bennett C H, DiVincenzo D P, Shor P W, Smolin J A, Trehal B M and Wootters W K 2001 Phys. Rev. Lett. 87 077902

[13] Zhan Y B 2005 Commun. Theor. Phys. 43637

[14] Huang Y X and Zhan M S 2004 Phys. Lett. A 327404

[15] Liu J M and Wang Y Z 2003 Phys. Lett. A 316159 